\title{
Práctica social, economía alternativa y espacios de proximidad en la ciudad de Valladolid
}

\author{
Social practice, alternative economy and spaces of proximity \\ in the city of Valladolid (Spain)
}

\author{
HENAR PASCUAL RUIZ-VALDEPEÑAS, ESTHER GIL ÁLVAREZ \& JUAN CARLOS \\ GUERRA VELASCO \\ Universidad de Valladolid. Departamento de geografía
}

Artículo recibido: 23 de enero de 2018

Solicitud de revisión: 12 de marzo de 2018

Artículo aceptado: 24 de mayo de 2018

\begin{abstract}
Pascual Ruiz-Valdepeñas, Henar; Gil Álvarez, Esther \& Guerra Velasco, Juan Carlos (2018). Práctica social, economía alternativa y espacios de proximidad en la ciudad de Valladolid. Recerca. Revista de Pensament i Anàlisi, 23, pp. 193-218.
\end{abstract}

Resumen

Durante la última década se ha producido un importante desarrollo de prácticas que formulan propuestas alternativas a los modos de producción, consumo e intercambio dominantes. Este artículo analiza el tejido de prácticas económicas alternativas en la ciudad de Valladolid, una ciudad media alejada de los contextos urbanos sobre los que se ha construido el cuerpo teórico que indaga en el potencial transformador de redes de consumo, mercados de trueque, bancos de tiempo, monedas sociales o huertos comunitarios. El trabajo, consciente del valor del contexto y de la escala, propone para ciudades como la estudiada una relectura que sitúa dicho potencial no en el ámbito económico, sino en el de la práctica y la experiencia social y en el de la cultura política.

Palabras clave: economía alternativa, proximidad, contexto urbano, espacio relacional y práctica social.

\begin{abstract}
The last decade has witnessed an important development of practices that involve alternative proposals to the dominant production, consumption and exchange patterns. In the present article, we present an analysis of the interwoven patterns of alternative economic practices in Valladolid, a mid-sized city in Spain. This urban profile differs from the cities that have typically been used to design the body of research on the transformation power that consumer networks, bartering, time banking, social coin and community orchards have. Taking into account the value of both the context and the scale under investigation, this study proposes a reanalysis for these types of cities: under this view, the potential is not
\end{abstract}


placed in the economic sphere but rather in the social practice and experience as well as in the political culture.

Keywords: alternative economy, proximity, urban context, relational space and social practice.

\section{INTRODUCCIÓN}

Las prácticas económicas alternativas (PEA) conforman un vasto conjunto de actividades, algunas de larga tradición (grupos de consumo agroecológico, bancos de tiempo, mercados de trueque y de productores, moneda social, tiendas gratuitas, huertos urbanos, centros sociales autogestionados, espacios de coworking,...) que conocen en la última década un importante desarrollo. En éste confluyen una genérica crítica al capitalismo, la insatisfacción con los modos de producción del espacio urbano y diversos factores relacionados con los efectos de la crisis económica y sus implicaciones en las ciudades y entornos metropolitanos.

El trabajo ${ }^{1}$ que se presenta aborda el estudio de un caso:Valladolid. Una parte de la literatura sobre las PEA tiene como materia de trabajo los artefactos sociales, económicos y espaciales que se construyen en ciudades donde la capacidad innovadora vinculada a lo no normativo constituye un elemento reconocible de su personalidad (Buttle, 2008; Paddeu, 2012; Conill et al., 2012; Vanolo, 2013; Tornaghi, 2014; Moulaert et al., 2014; Bresnihan \& Byrne, 2015; Psarikidou, 2015; Gonick, 2016; Fernàndez \& Miró, 2016; Michelini et al., 2017). Utilizando este cuerpo teórico, parece oportuno preguntarse por los atributos, las capacidades y el sentido que prácticas semejantes en lo nominal adquieren en contextos alejados de aquéllos que han servido para caracterizar su naturaleza alternativa y transformadora. El trabajo no reformula críticamente el sentido de esta nueva economía, sino que relee su alcance e indaga en la personalidad innovadora o adaptativa que en ese mismo contexto adquieren las prácticas que conforman este análisis.

1 Este artículo se encuadra en el proyecto de investigación «Espacios y prácticas económicas alternativas para la construcción de la resiliencia en las ciudades españolas» (2016-2018). Programa Estatal de Investigación, Desarrollo e Innovación Orientada a los Retos de la Sociedad, financiado por el Ministerio de Economía y Competitividad y el Fondo Europeo de Desarrollo Regional, referencia CSO2015-65452-R (Mineco/Feder).

Los autores queremos mostrar nuestro agradecimiento a los revisores de Recerca. Sus observaciones han sido fundamentales para mejorar el resultado final de este trabajo. 
Dadas las características organizativas de las PEA, la ausencia de referencias o estudios previos en la ciudad de Valladolid y la reducida literatura sobre otros ejemplos en España, este trabajo exigió en su desarrollo una estrategia metodológica cualitativa. El estudio parte de la identificación del tejido de prácticas alternativas. Tras la comprobación sobre el terreno de las iniciativas que se mantienen operativas, se realizaron entrevistas semiestructuradas a informantes significativos de las veinticuatro prácticas. Adicionalmente se realizaron encuestas cerradas, dirigidas a las personas implicadas en estos colectivos con el fin de conocer su perfil sociodemográfico e ideológico y su posición respecto a cuestiones relevantes para los objetivos de la investigación. Esta encuesta se realizó partiendo de una población de 1.600 participantes en las diferentes prácticas. La muestra se articuló buscando la aleatoriedad a pie de actividad, en diferentes franjas horarias e intentando evitar sesgos muestrales. Con este planteamiento fueron encuestadas 160 personas. A pesar del carácter cualitativo y exploratorio del estudio, las condiciones de significatividad son aceptables.

Los datos obtenidos se nutrieron con otras fuentes documentales: documentos de trabajo y de organización interna de los grupos, convenios de colaboración con la Administración local, actas de los encuentros de las redes, así como materiales de comunicación. El rastreo sistemático de los contenidos de las páginas web, blogs y redes sociales que utilizan como herramienta de formulación de propuestas, difusión de sus actividades y promoción de la participación, complementó la información y permitió asistir a distintos eventos (ferias, talleres, mercados) para conocer en el terreno el funcionamiento de los grupos.

Si se asume el valor del contexto histórico y geográfico en el funcionamiento y resultados de las PEA (Fickey \& Hanrahan, 2014), es necesario presentar el contenedor de todas ellas: la ciudad de Valladolid. Ésta puede ser definida dentro del sistema urbano español como una ciudad media por su tamaño y funciones. Rebasa en poco los 300.000 habitantes y en ella conviven la influencia de una importante tradición industrial junto con los cambios sociodemográficos derivados de su condición de oficiosa capital regional de Castilla y León. La tradición obrera se conserva de una manera visible en la morfología de la ciudad. El desarrollo que experimenta Valladolid entre mediados de la década de 1950 y toda la de 1960, junto con el carácter de emergencia que adquiere el crecimiento inmobiliario en ese intervalo, da lugar a espacios tan densamente poblados como escasamente equipados (García, 2000). En ellos se desarrolla un movimiento social que tiene en la asociación de vecinos su exponente más genuino y en la lucha 
por la mejora de los estándares urbanísticos su elemento aglutinador (Gonzalo, 2010 y 2011; Ruiz, 2013). Se configura de esta manera una periferia obrera con un fuerte componente vecinal y solidario (sobre todo en el norte y este de la ciudad) que durante años no sólo cultiva su dimensión reivindicativa, sino que también desarrolla proyectos comunitarios que tejen cada barrio y enraízan dinámicas. El significativo crecimiento superficial de Valladolid en los últimos treinta años, los trasvases de población entre barrios que lo sostienen y los cambios sociológicos que experimenta la ciudad se sobreimponen a la tradición antes reseñada. Surgen nuevas formas de lo urbano -la manzana abierta y la vivienda unifamiliar- que albergan sistemas de relaciones en los que lo comunitario, en consonancia con lo indicado por algunos autores (Spencer, 2015), se diluye. En este período, Valladolid pierde paulatinamente su carácter de banco de pruebas de políticas progresivas en lo social para, a partir de 1995, emerger un perfil más conservador en lo social.

\section{LAS PRÁCTICAS ALTERNATIVAS: UNA REVISIÓN DE SUS FUNDAMENTOS TEÓRICOS}

La abundante literatura existente relativa a las prácticas alternativas comparte toda ella una característica: su amplitud conceptual. Su revisión, en sintonía con las aportaciones pioneras de Gibson y Graham (2008), permite encuadrarlas en el contexto de las economías diversas, diferentes o alternativas que, relegadas en la mercantilización pero emergentes en la recesión, cuestionan con desigual intensidad el modelo capitalista imperante, demandan fórmulas alternativas y manifiestan cierta desconfianza hacia la clase política dirigente (Healey, 2009; Jones \& Murphy, 2011). Su diversidad y variedad dificulta su delimitación, siendo el resultado de un sinfín de corrientes y movimientos sociales que, con diversos matices y antigüedades, se reactivan criticando el sistema capitalocentrista neoliberal, oponiéndose a él, conviviendo o tratando de superarlo (Leyshon et al., 2003; Gritzas \& Kavoulakos, 2015).

Su tipificación se asocia a criterios básicos relacionados con su organización en redes de colaboración, su adscripción a la máxima de la solidaridad, su condición de alternativas y su proximidad geográfica, pudiendo sistematizarse en cuatro tipos básicos dependiendo de su función en el circuito económico: producción de bienes y servicios, intercambio, consumo colaborativo y finanzas alternativas con arraigo territorial (Méndez, 
2015). Bajo estas etiquetas se incluye un grupo de actividades que abarcan desde la economía informal, hasta las diferentes organizaciones encuadradas en la economía social y el llamado tercer sector, cercanas a la economía convencional sin ánimo de lucro (Sánchez, 2017: 69). Sus miembros suscriben los principios de autonomía, reciprocidad y democracia, promueven valores no competitivos (solidaridad, sostenibilidad, cooperación, equidad, etc.) y actúan en un ámbito local con espacios físicos de encuentro colectivo (Sánchez, 2017: 43). Desde el prisma motivacional, la prioridad concedida al valor de uso de los bienes y servicios y la voluntad de densificar los vínculos sociales son algunos de los argumentos esgrimidos en sus discursos y acciones, particularmente en el caso de los actores más concienciados e implicados (Conill et al., 2012). Desde el prisma de su estructura organizativa, predomina la red, con relaciones de reciprocidad múltiples generadoras de capital social y relacional frente al individualismo inherente al modelo económico imperante (Cardoso \& Jacobetty, 2013).

Por otro lado, el vínculo con el territorio y la valorización de sus recursos introducen una dimensión espacial generalmente ignorada en la literatura científica o limitada a alusiones puntuales sobre su localización. Sin embargo, resulta evidente que las actividades se dan en determinados lugares y momentos por motivos concretos y que estas características espaciotemporales favorecen su proliferación o frenan su desarrollo. De ahí, la coincidencia con North (2005) al esgrimir que, en la génesis de prácticas, la escala es importante. Finalmente, es necesario mencionar los obstáculos y debilidades que las afectan. Son cuestiones objeto de atención la escasez de los recursos materiales y el tamaño de los grupos, en cuanto amenazas potenciales para su supervivencia, o la calidad del empleo generado y su integración en el mercado laboral formal (Méndez, 2015: 17).

El trabajo que se presenta comparte por tanto la definición de estas economías alternativas como el resultado de unas prácticas situadas en las periferias del capitalismo o directamente fuera de éste por su carácter no lucrativo, no remunerado, no jerárquico y que se sustentan en estrategias locales de desarrollo comunitario y participativo. Si la escala local y la proximidad (Leyshon et al., 2003) dan sentido a su personalidad alternativa, cabe preguntarse por la capacidad transformadora que esas prácticas poseen en determinados contextos espaciales. Desde una perspectiva geográfica, la contextualidad y la escala se revelan como elementos indispensables en el estudio de las PEA. Responder a estos interrogantes, contribuir al debate sobre el valor del contexto urbano y su significado en la personalidad transformadora o adaptativa de estas prácticas, exige conocer el 
perfil y las motivaciones de quienes las promueven, así como los radios de acción y las estrategias organizativas y de funcionamiento que despliegan.

\section{LA IDENTIFICACIÓN DE LAS PRÁCTICAS ECONÓMICAS ALTERNATIVAS EN VALLADOLID}

De forma análoga a lo ocurrido en otros contextos urbanos, en Valladolid se ha producido un importante incremento de estas iniciativas como respuesta ciudadana a los efectos de la crisis económica que agudizó las desigualdades sociales y generó un fuerte cuestionamiento del modelo institucional y del sistema neoliberal dominante. Si atendemos al universo de prácticas -actualmente veinticuatro iniciativas cuya tipología se recoge sintéticamente en la tabla 1-, tres de ellas son anteriores a ese contexto de crisis, pero las demás surgen a partir de 2009.

La naturaleza de actores incluye asociaciones de larga tradición vecinal, organizaciones sindicales, pequeños colectivos ciudadanos autoorganizados, movimientos de transición y plataformas asamblearias del 15M.

La mayor parte de los huertos urbanos no posee finalidad productiva, ni siquiera autoconsumo. Su objetivo es la producción de espacio público relacional con el trabajo hortelano y el consumo compartido de la cosecha. Entrehuertos y De Vuelta a la Huerta además hacen entregas de sus productos a la despensa solidaria del proyecto social Entrevecinos, mientras que el BAH funciona mediante un sistema de reparto de cestas a los socios.

Los cuatro centros sociales autogestionados sirven de lugar de encuentro de colectivos y movimientos sociales. Con diferentes perspectivas y distintos grados de radicalidad, comparten una postura crítica con el orden político, económico, social y cultural vigente. Organizan de forma regular propuestas culturales alternativas, debates, talleres e intervenciones con la finalidad de producir un espacio político en el que trabajar una conciencia crítica y transformadora. 
Tabla 1

Prácticas económicas alternativas en la ciudad de Valladolid

\begin{tabular}{|c|c|c|c|c|c|}
\hline & Prácti & & Nombre & $\begin{array}{c}\text { Promotores } \\
\text { / Actores }\end{array}$ & $\begin{array}{c}A \tilde{n} o \\
d e\end{array}$ \\
\hline & & & $\begin{array}{l}\text { Bajo el Asfalto } \\
\text { está la Huerta }\end{array}$ & Colectivo Ван & 2006 \\
\hline & & & $\begin{array}{l}\text { La Huerta sin } \\
\text { Puerta }\end{array}$ & $\begin{array}{l}\text { colectivo } \\
\text { ciudadano }\end{array}$ & 2012 \\
\hline & & & $\begin{array}{l}\text { El Huertillo de } \\
\text { Pajarillos }\end{array}$ & Red Pajarillos & 2013 \\
\hline & & Huertos & $\begin{array}{l}\text { La Huerta de la } \\
\text { Granja }\end{array}$ & $\begin{array}{l}\text { colectivo } \\
\text { ciudadano }\end{array}$ & 2013 \\
\hline & $\begin{array}{l}\text { espacio } \\
\text { público }\end{array}$ & $\begin{array}{c}\text { urbanos } \\
\text { comunitarios }\end{array}$ & $\begin{array}{l}\text { De Vuelta a la } \\
\text { Huerta }\end{array}$ & $\begin{array}{l}\text { colectivo } \\
\text { ciudadano }\end{array}$ & 2013 \\
\hline & & & $\begin{array}{l}\text { La Huerta de } \\
\text { Belén }\end{array}$ & $\begin{array}{l}\text { A.V. } 24 \text { de } \\
\text { diciembre }\end{array}$ & 2014 \\
\hline Producción & & & Entrehuertos & $\begin{array}{c}\text { Proyecto } \\
\text { social } \\
\text { Entrevecinos }\end{array}$ & 2014 \\
\hline & & & $\begin{array}{l}\text { Gerihuerto de } \\
\text { Parquesol }\end{array}$ & $\begin{array}{l}\text { colectivo } \\
\text { ciudadano }\end{array}$ & 2015 \\
\hline & & & Las Dagas & $\begin{array}{l}\text { colectivo } \\
\text { ciudadano }\end{array}$ & 2009 \\
\hline & & & $\begin{array}{l}\text { Vientos del } \\
\text { Pueblo }\end{array}$ & $\begin{array}{l}\text { colectivo } \\
\text { político }\end{array}$ & 2014 \\
\hline & $\begin{array}{l}\text { espacio } \\
\text { político }\end{array}$ & $\begin{array}{c}\text { sociales } \\
\text { autogestionados }\end{array}$ & El Corral & $\begin{array}{l}\text { Colectivo La } \\
\text { Gallinera } \\
-15 \mathrm{M} \\
\text { Parquesol }\end{array}$ & 2014 \\
\hline & & & La Ortiga & $\begin{array}{l}\text { colectivo } \\
\text { ciudadano }\end{array}$ & 2016 \\
\hline
\end{tabular}




\begin{tabular}{|c|c|c|c|c|}
\hline \multicolumn{2}{|c|}{ Práctica } & Nombre & $\begin{array}{l}\text { Promotores } \\
\text { / Actores }\end{array}$ & $\begin{array}{c}A \tilde{n} o \\
d e\end{array}$ \\
\hline \multirow{9}{*}{ Consumo } & \multirow{9}{*}{$\begin{array}{l}\text { Redes de } \\
\text { consumo }\end{array}$} & La Patata Solidaria & $\begin{array}{l}\text { colectivo } \\
\text { ciudadano }\end{array}$ & 1997 \\
\hline & & $\begin{array}{l}\text { Ecogermen, S. } \\
\text { Cooperativa }\end{array}$ & $\begin{array}{l}\text { red de } \\
\text { consumo }\end{array}$ & 2009 \\
\hline & & La Cesta Verde & $\begin{array}{l}\text { colectivo } \\
\text { ciudadano }\end{array}$ & 2012 \\
\hline & & El Repollo Mutuo & CNT & 2012 \\
\hline & & $\begin{array}{l}\text { La Endivia } \\
\text { Cochina }\end{array}$ & CGT & 2012 \\
\hline & & $\begin{array}{l}\text { El Caracol de } \\
\text { Parquesol }\end{array}$ & $\begin{array}{l}\text { Colectivo La } \\
\text { Gallinera } \\
-15 \mathrm{M} \\
\text { Parquesol }\end{array}$ & 2013 \\
\hline & & La Lenteja Pelleja & $\begin{array}{l}\text { 15M La } \\
\text { Rubia }\end{array}$ & 2013 \\
\hline & & El Ajo en Red & $\begin{array}{c}\text { red de } \\
\text { consumo }\end{array}$ & 2013 \\
\hline & & $\begin{array}{l}\text { La Despensa del } \\
\text { Tío Chaqueta }\end{array}$ & $\begin{array}{l}\text { colectivo } \\
\text { ciudadano }\end{array}$ & 2014 \\
\hline \multirow{3}{*}{ Intercambio } & $\begin{array}{l}\text { Bancos de } \\
\text { tiempo }\end{array}$ & $\begin{array}{l}\text { Banco del tiempo } \\
\text { de Valladolid }\end{array}$ & $\begin{array}{l}\text { Ayuntamiento } \\
\text { de Valladolid }\end{array}$ & 2005 \\
\hline & $\begin{array}{c}\text { Mercados de } \\
\text { trueque }\end{array}$ & Tejiendo Redes & $\begin{array}{c}\text { Tejiendo } \\
\text { Redes de } \\
\text { Participación } \\
\text { Social - 15M } \\
\text { Delicias }\end{array}$ & 2011 \\
\hline & $\begin{array}{l}\text { Monedas } \\
\text { sociales }\end{array}$ & Vecino & $\begin{array}{c}\text { Proyecto } \\
\text { Social } \\
\text { Entrevecinos }\end{array}$ & 2011 \\
\hline
\end{tabular}

Fuente: elaboración propia 
Las redes de consumo, integradas por entre quince y veinte unidades familiares que configuran su propia red de abastecimiento, responden al modelo de canal corto de comercialización de productos agroecológicos. $\mathrm{Su}$ ideario se basa en principios éticos y solidarios con el mundo rural, la sostenibilidad ambiental y la soberanía alimentaria. Los grupos funcionan de manera similar, si bien Ecogermen optó por profesionalizar la gestión y constituirse en sociedad cooperativa de consumo ecológico en 2009, contando con personal contratado y tienda propia.

El Banco del Tiempo es de iniciativa municipal y opera como agente de intercambio vecinal de servicios mediante el uso del tiempo como unidad de valor. Son intercambios multirrecíprocos, individuales o grupales que no requieren profesionalización. Su objetivo es activar redes de cooperación y solidaridad en la comunidad y crear espacios de encuentro como herramienta para trabajar la integración social. El ámbito territorial es el barrio de La Victoria, pero está abierto a la participación de toda la ciudad.

El mercado de trueque que se mantiene activo es el vinculado a Tejiendo Redes, colectivo de acción social que promueve la implicación de los vecinos del barrio de Las Delicias. El tipo de trueque es directo y el mercado se organiza durante una jornada en el espacio público. A la finalidad de resolver las necesidades de las personas sin mediar transacciones monetarias, se une el objetivo más amplio de trabar redes comunitarias en el barrio.

Finalmente, la experiencia de la moneda social Vecino se circunscribe al proyecto social Entrevecinos, localizado en el barrio de España. El objetivo del proyecto es la orientación y la inserción laboral y cuenta además con una despensa solidaria donde los productos se adquieren mediante la moneda social que se facilita a cada usuario a cambio de su trabajo activo en la búsqueda de empleo.

\section{LA PRÁCTICA DE LAS PRÁCTICAS ECONÓMICAS ALTERNATIVAS}

3.1. La construcción de nuevas lógicas de colaboración y la formulación de alternativas

El sistema de valores en el que la solidaridad prima sobre el interés individual o el beneficio material (Bioteau \& Fleuret, 2014), adopta formas distintas en los discursos de las prácticas analizadas. Expresiones como 
sacar adelante un proyecto común, sentimiento comunitario, juntar gente con ideas en común, o trabajar de forma colectiva, aparecen como argumento recurrente: lo colectivo o lo comunitario es lo que da sentido a las lógicas de las prácticas. Sobre estos principios se fundamenta una acción con objetivos transformadores que surge de formas alternativas de sociabilidad basadas en la confianza y la reciprocidad (Bresnihan \& Byrne, 2015; Méndez, 2015; Conill et al., 2012). En los centros sociales autogestionados se trabaja el autoconocimiento personal y la cohesión del grupo. En los huertos urbanos comunitarios prevalece el deseo de favorecer la creación de tejido social y el fortalecimiento de los vínculos vecinales. Los integrantes de los grupos de consumo otorgan un notable valor al trabajo colectivo y al compromiso personal que de él se deriva. En el mercado de trueque se persigue promover la implicación del barrio en actividades destinadas a estrechar lazos y fortalecer el sentimiento comunitario. El Banco del Tiempo añade a su funcionamiento cotidiano, el objetivo de activar redes de cooperación y solidaridad en la comunidad.

La posición frente al sistema capitalista dista de ser común y conviven posiciones y formulaciones diferenciadas. Por una parte, las que se plantean como iniciativas de resistencia y ponen el acento en proyectos militantes para la formación de una conciencia crítica radical y la creación de «espacios liberados» en los que cristalice una forma de vida contrahegemónica a través de procesos de transformación personal (La Ortiga, BAH, Vientos del Pueblo). Por otra, iniciativas cuya práctica consideran que contribuye a corregir los aspectos más injustos del sistema. Es el caso del grueso de los huertos urbanos «trabajan por hacer más sostenible el actual modelo de ciudad, diseñar un paisaje urbano diferente, crear espacios abiertos a la participación y potenciar el tejido social» y de los grupos de consumo, que se enfrentan al sistema alimentario corporativo con criterios agroecológicos y sociales (Watts et al., 2005; Psarikidou, 2015).

El perfil ideológico puede estar vinculado a una cultura política que se aprende con la propia práctica y el espacio concreto en el que ésta tiene lugar. El csa Vientos de Pueblo ocupa el local que históricamente albergó la sede del PCPE y las reuniones y actividades de la izquierda revolucionaria en la ciudad; por su parte, los grupos de consumo Endivia Cochina y Repollo Mutuo nacen vinculados a los sindicatos CGT y CNT respectivamente y comparten local con estas organizaciones anarcosindicalistas y libertarias.

No obstante, también aparecen prácticas cuya capacidad para cuestionar el orden establecido se ha desdibujado con el tiempo, principalmente por la reducción del tamaño del grupo. El Gerihuerto de Parquesol, la Huer- 
ta de la Granja y el вAн mantienen las motivaciones transformadoras de base, pero la potencialidad de su enfoque se encuentra debilitada respecto a su fuerza inicial. De hecho, en estos pequeños grupos es evidente una de las limitaciones que, de orden general, se han resaltado en estas iniciativas: su pequeño tamaño (Conill et al., 2012: 308). Éste, si bien facilita las relaciones personales, dificulta la prestación de servicios o el acceso a los bienes implícitos a cada actividad.

\subsection{Las redes y la proximidad organizativa}

Las prácticas económicas alternativas adoptan diversas formas de organización, pero el análisis de estas experiencias muestra que predominan los colectivos sin formalización jurídica (figura 1). Se trata de fórmulas organizativas que rechazan las estructuras jerárquicas y trabajan en espacios autogestionados mediante relaciones horizontales de proximidad, asamblearias, colaborativas y deliberativas.

La proximidad organizativa también tiene su reflejo en la ordenación de las tareas cuyo criterio de funcionamiento es la reciprocidad y la corresponsabilidad. Una abrumadora mayoría de las prácticas funcionan con trabajo voluntario rotatorio. No obstante, en este marco de flexibilidad es posible identificar algunas diferencias en función de la modalidad de participación. En las prácticas sujetas a admisión, las tareas son asumidas periódicamente por todos los miembros de la red como parte de su compromiso. En cambio, en las prácticas que funcionan con modalidades abiertas de participación la planificación de los trabajos la realizan quienes tienen mayor disponibilidad o estabilidad en el grupo, pero las tareas que se acometen periódicamente, son asumidas de forma completamente libre entre los participantes, sin turnos ni compromisos, más allá de un horario de encuentro (La Huerta sin Puerta, La Huerta de la Granja, El Huertillo de Pajarillos, La Huerta de Belén, Gerihuerto de Parquesol). Otras, como el ван, presentan formas más articuladas de organización con diversos espacios de participación. Cuentan con un grupo «liberado» y tres grupos de consumo cuyo cometido es realizar los repartos entre los socios. 


\section{Figura 1}

Criterios organizativos, de funcionamiento y evolución temporal de las prácticas económicas alternativas en la ciudad de Valladolid (1997-2017)
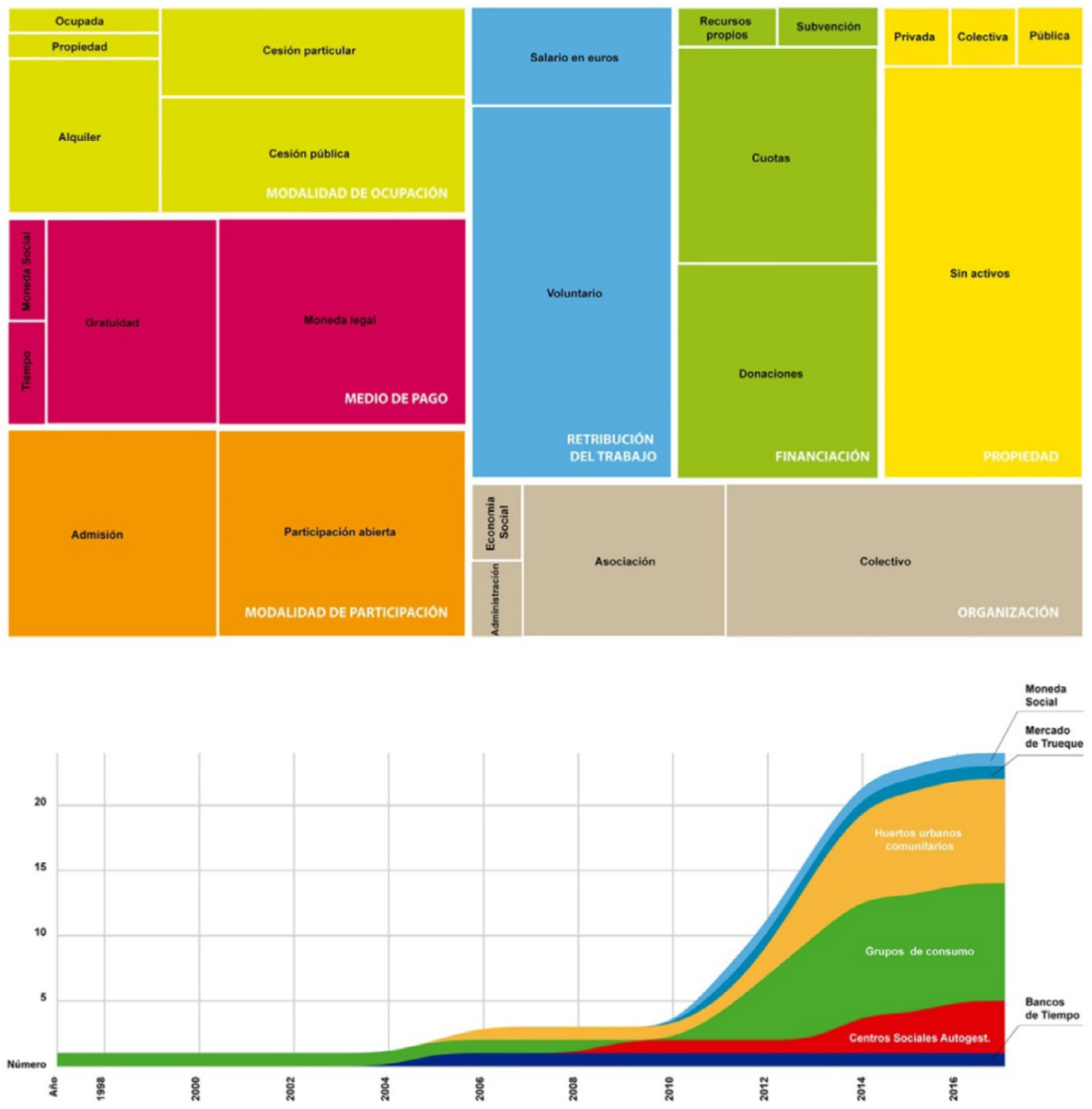

Fuente: elaboración propia.

Comúnmente las dinámicas organizativas se definen con independencia de la naturaleza jurídica de los grupos. Es decir, el hecho de registrarse como asociaciones no supone diferencias significativas sino que está relacionado, bien con el origen de la iniciativa a partir de asociaciones vecinales o bien con 
la necesidad de gestionar más fácilmente cuestiones como la firma del contrato del suministro eléctrico, el alquiler del local o los permisos municipales para la ocupación de espacios públicos. En cualquier caso, en los discursos se subraya siempre la idea de mantener su autonomía frente al orden normativo vigente. No poseen ningún tipo de dependencia respecto de ayudas públicas ya que su financiación proviene de recursos propios en forma de aportaciones voluntarias de los participantes o cuotas de los socios. Su relación con la Administración local ha sido durante años prácticamente nula o incluso puntualmente conflictiva. Sólo tras la llegada en 2015 de un nuevo equipo de gobierno municipal, se ha producido una normalización institucional en las relaciones con estas redes. Es lo que sucede con algunos huertos como La Huerta sin Puerta y el Huertillo de Pajarillos que, en origen, ocuparon parcelas de titularidad pública y han «formalizado» la situación mediante la firma de convenios de colaboración para su ocupación en precario y gratuita.

\subsection{Los aprendizajes colectivos como semillero de prácticas alternativas}

Si la proximidad organizativa constituye uno de los rasgos de identidad de las prácticas alternativas, los actores implicados en ellas son capaces de crear espacios de aprendizaje. El grupo se convierte en un lugar creativo de generación de propuestas en el que se comparten y se integran las ideas. Su fuerza reside en compartir saberes y habilidades que dan lugar a nuevas formas de aprendizaje colectivo.

Bajo estos principios se organizan las tareas en los huertos comunitarios, en los cuales participan mayoritariamente personas sin conocimientos hortícolas previos. Las entrevistas revelan la forma en la que comparten y transmiten los saberes. Más allá de la presencia de personas con formación específica, todos los grupos coinciden en señalar que los conocimientos se adquieren con la práctica, se comparten entre las huertas, se generan en las redes y se conforman con las ideas de todos, incluyendo a los vecinos del barrio que no participan directamente en la huerta pero que se acercan a conocerla. Por su parte, los centros sociales autogestionados, como La Ortiga, trabajan activamente en la idea de equiparar saberes y conocimientos; de integrar conocimientos segmentados y desconectados; muestran su rechazo a la formación reglada y construyen un espacio en el que rige un modelo de vida alternativo basado en el autoconocimento y la ética del cuidado mutuo De alguna forma, también las redes de consumo comparten estos argumentos. Todos los grupos manifiestan su empeño en 
la creación de espacios de formación y reflexión sobre soberanía alimentaria, justicia socioambiental o criterios de compra ética. Sin embargo, también expresan abiertamente las dificultades a las que se enfrentan para mantener activas estas acciones, debido en ocasiones al reducido tamaño del grupo, la falta de tiempo o el consumo de las energías en las tareas habituales para el funcionamiento de la red.

En cierto modo, estos mismos factores son los que limitan las posibilidades de generar alianzas duraderas entre las prácticas alternativas. Existen experiencias de cooperación entre los diversos colectivos que articulan redes informales para trabajar en objetivos comunes. Esos vínculos pueden ser el resultado de decisiones grupales o bien pueden surgir espontáneamente de las personas que están comprometidas con más de un grupo y que ejercen de nodo de conexión entre las distintas prácticas. Los resultados de estos procesos colaborativos a veces son meros acuerdos entre las redes para la gestión integrada de pedidos conjuntos. Pero también pueden plantearse con objetivos más amplios, como sucede con la red de huertos comunitarios de la ciudad, la celebración de encuentros entre las redes de consumo o las actividades compartidas por los centros sociales autogestionados. Sin embargo, el alcance de estas experiencias es todavía limitado.

La proximidad cognitiva y el factor relacional entre individuos vinculados por el entusiasmo y la tenacidad otorgan a estas prácticas capacidad para replicar modelos: las prácticas alternativas se convierten en semilleros de nuevas iniciativas. Por ejemplo, de la red de consumo más antigua surgieron otros proyectos de consumo con distintos planteamientos; la experiencia de las primeras huertas comunitarias se replicó en casi todos los barrios de la ciudad; de las asambleas del 15M brotaron distintos colectivos, de los cuales nacieron, a su vez, otros proyectos de perfil autogestionado; en La Huerta sin Puerta germinó la compañía de teatro del mismo nombre.

Los aprendizajes compartidos rompen con el paradigma imperante en la adquisición de conocimiento, a la vez que permiten ampliar la proyección de estas prácticas. Rebasan el espacio de los grupos para conectar con el tejido asociativo y los movimientos sociales de la ciudad a través de la integración en proyectos de barrio más amplios, transversales y comprometidos.

\section{LA BASE SOCIAL DE LAS REDES ALTERNATIVAS}

Para identificar el perfil de los grupos sociales que impulsan y activan el tejido de prácticas alternativas, es preciso hacer una aproximación al tamaño 
de las redes. A partir de la información obtenida en las entrevistas, los criterios de recuento están condicionados por su carácter de redes dinámicas en constante transformación. Exceptuando el Banco del Tiempo y Ecogermen, que, en el momento de la toma de datos, contaban con un registro de 617 y 108 socios respectivamente, es complicado determinar con precisión el número de personas implicadas en estos colectivos. Para las redes de consumo, en las que el número de unidades familiares varía con relativa frecuencia, se pudo estimar una cifra total de 550 personas. En el resto de prácticas, que funcionan con estructuras informales abiertas, el recuento supera las 300 personas y se refiere a quienes mantienen un vínculo estable o no con la red. Por tanto, aunque es una cifra orientativa, es posible calcular que las prácticas alternativas aglutinan conjuntamente a unos 1.600 participantes.

Su perfil sociodemográfico es el de una persona de mediana edad ( 47 años) con estudios superiores (68\%), con trabajo y algún tipo de activismo previo. El rango de edad de quienes conviven en los grupos es amplio, pero se detectan patrones contrastados, apreciables sobre todo entre los centros sociales autogestionados, cuyos integrantes son mayoritariamente jóvenes menores de 35 años, y los huertos comunitarios, en los que predominan las personas mayores de 50. El 82 \% son trabajadores en activo y las ocupaciones más frecuentes son las de la enseñanza, la educación y el trabajo social, los trabajos técnicos, los puestos administrativos y las profesiones del sector sanitario. Casi la mitad (48\%), forman parte de unidades familiares que disponen de un umbral de ingresos (20.00040.000 euros) igual o superior a la renta media nacional (26.730 euros en 2016) y el tamaño medio de los hogares es de 2,6 personas (figura 2). No se trata, por tanto, ni de grupos sociales vulnerables ni de colectivos de jóvenes sino de grupos de estatus social medio y nivel de cualificación alto.

Quienes forman parte de estas prácticas han conocido su existencia a través de amigos (60\%) y su incorporación se ha producido a partir del año 2012 (81\%): el clima de movilización ciudadana que se origina en 2011 estimula el proceso de agregación de participantes en los años posteriores, atraídos por su carácter alternativo e innovador en la ciudad.

El análisis de los motivos que impulsan a la participación revela que los más importantes están asociados a la idea de contribuir a mejorar la sociedad y cuestionar la economía convencional. Las posturas se decantan por formulaciones para la corrección de los aspectos más injustos del sistema capitalista, posición con la que se identifica la mitad de los encuestados. El rechazo total del capitalismo como sistema económico y social es compartido por el $27 \%$ de los participantes mientras que el 23\% eligen construir unas relaciones económicas que les permitan vivir completamente al margen del sistema. 


\section{Figura 2}

Resultados de la encuesta a los participantes en las prácticas económicas alternativas en la ciudad de Valladolid

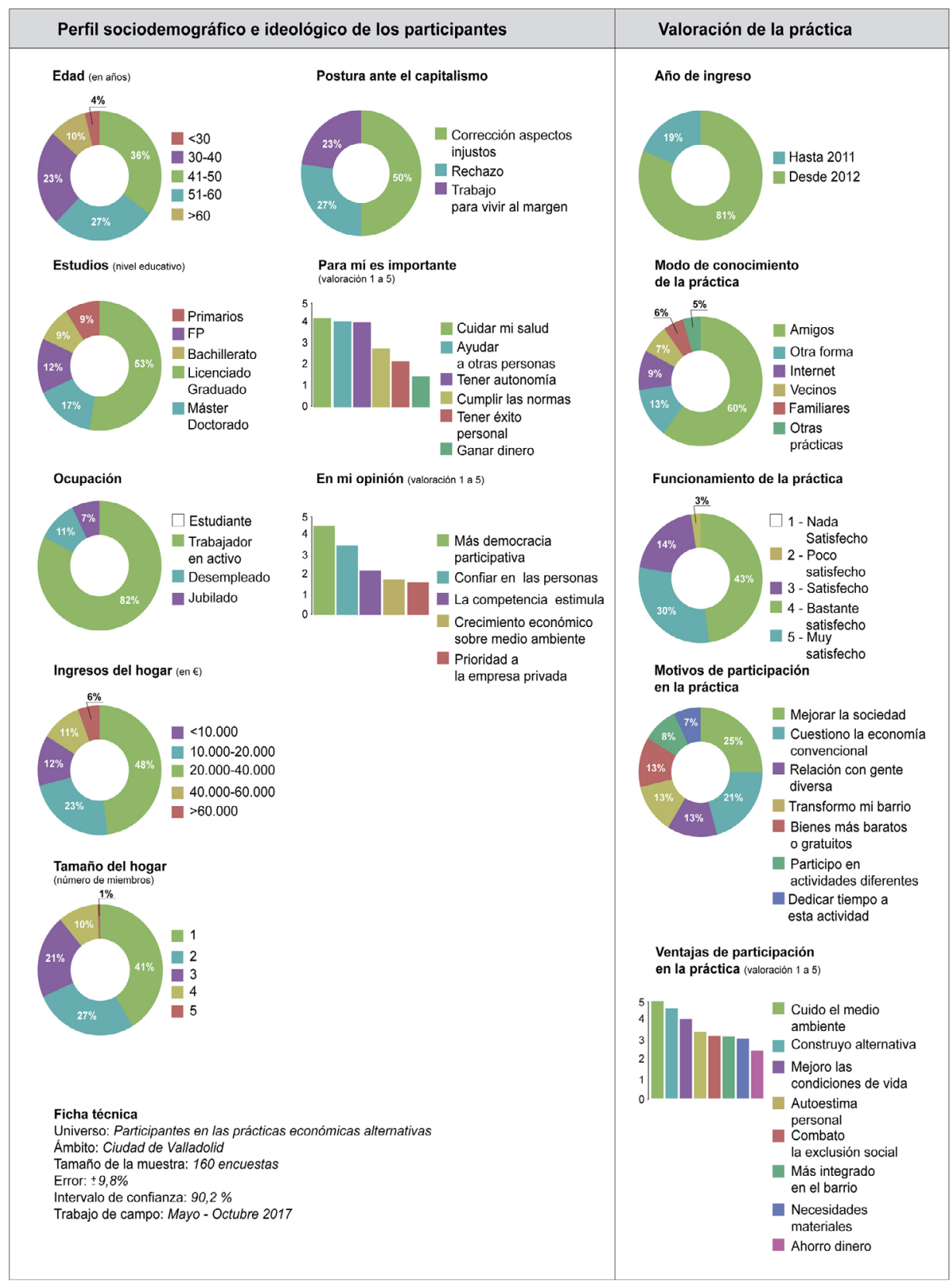


Respecto a los valores vitales a los que se atribuye mayor importancia, el cuidado de la salud y la alimentación aparece en primer término. La autonomía y la iniciativa personal para implicarse en proyectos colectivos con creatividad, responsabilidad y sentido crítico, es igualmente un aspecto prioritario para quienes trabajan en las redes, al igual que el valor ético de la solidaridad.

Por lo que se refiere a las opiniones acerca de la organización económica y política y el sistema de relaciones sociales, se sitúa en primer término la necesidad de desarrollar formas de democracia participativa que faciliten una influencia directa en la elaboración de políticas públicas. También se confiere un gran valor a la interacción social, a la capacidad de establecer relaciones de confianza compartiendo y colaborando en actividades grupales. Cuestión fundamental es además la consideración de categorías emocionales como la mejora de la autoestima personal, entendida como resultado de un proceso de fortalecimiento de la confianza al sentirse parte de un colectivo capaz de impulsar transformaciones tangibles en su contexto social y territorial.

En sintonía con este proceso de empoderamiento personal y grupal, el nivel de satisfacción con el funcionamiento de la práctica es bastante elevado. Eso no supone, sin embargo, una actitud acrítica con el grupo. Las reflexiones personales en este sentido enfatizan la necesidad de reforzar el nivel de compromiso, trabajar por la agregación vecinal y fortalecer las capacidades de la red mediante una mayor apertura, vinculación y transversalidad con otros grupos.

En la densidad como experiencia de las PEA desempeña un papel significativo la cultura económica que aportan quienes participan en ellas. Otros estudios (Conill et al., 2012: 129) han puesto de manifiesto la existencia de tres grados decrecientes de convencimiento alternativo: personas culturalmente transformadoras, personas que comparten estas prácticas, pero no le asignan un significado disruptor y personas culturalmente adaptadas al modelo hegemónico. Las respuestas de los cuestionarios muestran la presencia de los tres grupos en el caso estudiado, pero también evidencian el predominio de aquellas alejadas de planteamientos que implican una activa ruptura cultural con el capitalismo. 


\section{ESPACIO URBANO Y PRÁCTICAS ECONÓMICAS ALTERNATIVAS: EL VALOR DE UN ESPACIO RESISTENTE Y AUTÓNOMO}

Frente a la tipificación de las prácticas, de su funcionamiento interno o del sistema de valores que las informan, las relaciones que establecen con el espacio urbano no han sido un objeto de atención preferente en la literatura especializada. La imagen de resistencia social que la historia reciente de la ciudad ha proporcionado a ciertos barrios de Valladolid tiene también su reflejo en el discurso de las prácticas. Si se reside o se participa de alguna manera en la vida de un espacio de tradición militante -con independencia de la intensidad con la que se exterioriza en la actualidad- no pueden faltar aquellas manifestaciones sobre las que hoy se construye la personalidad creativa e innovadora de un barrio o la expresión social de la movilización de carácter alternativo (García et al., 2016; Sánchez et al., 2017). No obstante, en Valladolid la tradición militante no desborda los habituales cauces de expresión de la reivindicación vecinal, social y política. No ha creado ámbitos, como ocurre en otras ciudades españolas, connotados por la ruptura con el sistema. Si bien esta lucha existe en Valladolid, ejemplificada por algunas ocupaciones de edificios con escaso recorrido temporal, no posee ni la masa, ni el grado de articulación, ni las capacidades suficientes como para construir nuevas utopías, espacios o lógicas alternativas a las ya identificadas y normalizadas socialmente como tales.

Las prácticas analizadas surgen en contextos urbanos y, con independencia de sus características particulares o de los discursos complementarios que cada una maneja, suponen una respuesta al modelo de ciudad capitalista imperante (Nel·lo, 2015). Reubican la función de socialización contenida en todo espacio público de la ciudad: salta de la plaza y la calle a los nuevos espacios de proximidad que las prácticas crean, ya sea el huerto urbano, la red de consumo o el centro social autogestionado. A su vez, incorporan la noción de bien común (Mattei, 2013; Abad \& Abad, 2014; Felber \& Hagelberg, 2017), pero con ella no sólo se alude al suelo urbano, sobre todo aquel de propiedad pública y expectante de uso, sino también a valores inmateriales «la alimentación» o flujos de energía «metabolismo urbano».

Junto con la autonomía social, quizá el rasgo que mejor define espacialmente la dimensión de las prácticas alternativas es la cercanía. Constituye, desde diversas perspectivas «económica, geográfica, social y afectiva» y con diversas capas, uno de sus rasgos más personales. A través del valor que se 
proporciona a lo local se busca la recuperación de la proximidad (Bioteau \& Fleuret, 2014). Es ésta, con los lazos íntimos que teje, la que construye personas con capacidad de resistir y crear (Esquirol, 2015).

La escala del Banco del Tiempo y de la moneda social Vecino es ciertamente municipal. En el primero, por ser un proyecto de la Administración local que tiene ese ámbito como referencia, y el segundo, por las propias características del proyecto en el que se encuadra la moneda social. Una reflexión similar se puede realizar de los mercados de trueque. Como tal, sólo existe uno en la ciudad de Valladolid y el carácter informal y líquido de la participación en él hace difícil profundizar en su análisis, más allá de señalar que sus usuarios son vecinos del barrio (figura 3).

\section{Figura 3}

Lugar de residencia, por distrito postal, de los participantes en tres tipos de prácticas en el municipio de Valladolid

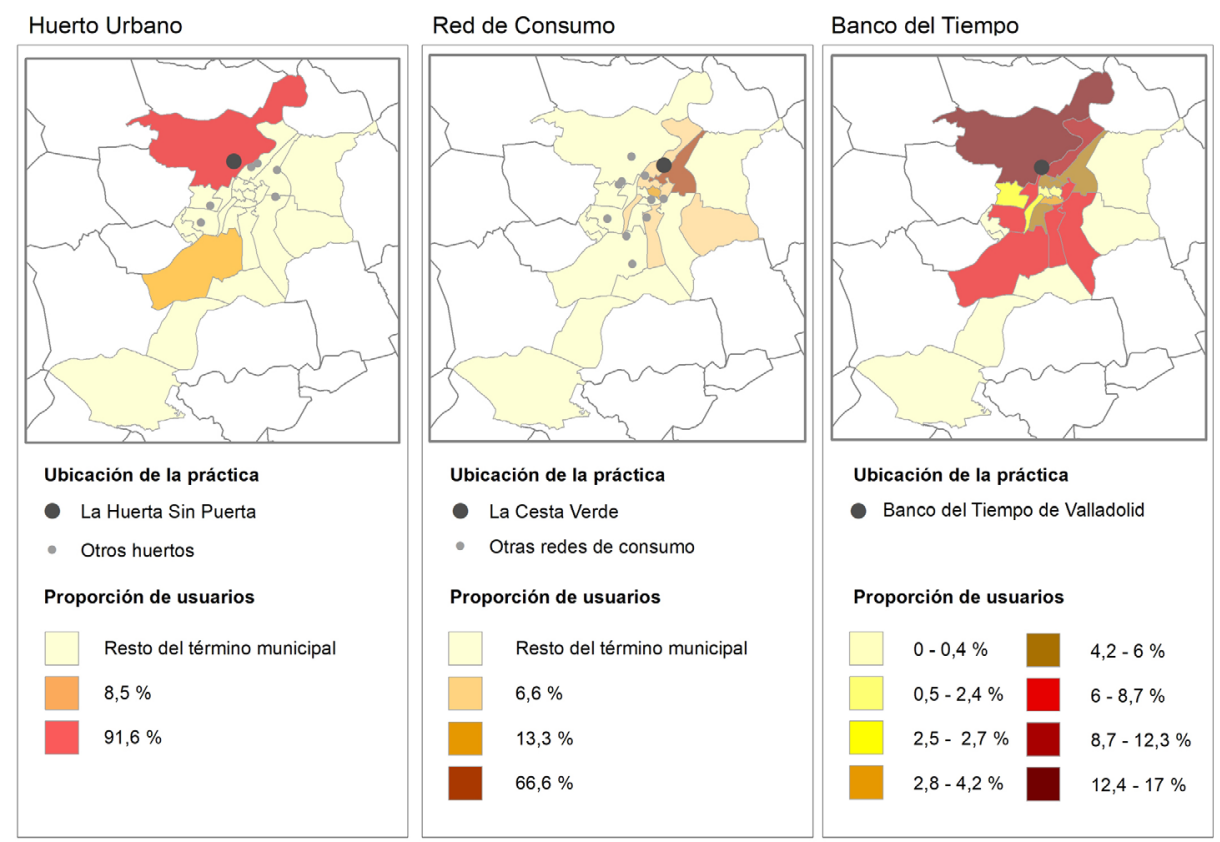

Fuente: Elaboración a partir de datos de encuesta 
La eclosión de redes de consumo en Valladolid a partir de 2012 y los encuentros que entre ellas realizan en los primeros años, dan lugar a una reflexión interna sobre su espacialidad. Se ven a sí mismas vinculadas a barrios concretos, en una malla con capacidad para atender a cualquier vecino sin necesidad de realizar un desplazamiento importante. Este modelo, aunque de alguna forma se mantiene, solo funciona en parte. El nomadismo en la búsqueda de un local apropiado para hacer los repartos hace que las redes muden de ubicación y que en este trasiego se produzca un balance difícil de cuantificar entre altas y bajas que, no obstante, deja espacios sin cubrir y sin usuarios que repongan la red desaparecida. Donde la fidelidad al espacio se mantiene de una manera permanente es, por su propia naturaleza, en los huertos urbanos comunitarios. No nacen con un fin productivo, sino que son entendidos como espacios relacionales que adquieren sentido dentro de la vivencia del barrio.

La espacialidad de las prácticas puede ser entendida desde el punto de vista de las relaciones funcionales que establecen entre ellas (Conill et al., 2012). En la construcción de una alternativa fundamentada en lo local y la cercanía, el engarce entre prácticas permitiría eludir o retrasar considerablemente la participación en las prácticas de mercado más convencionales. El huerto comunitario y la red de consumo abastecen al núcleo familiar -sin que este término tenga una sentido cultural-, el cual accede a otros bienes y servicios en un vector que encadena el mercado de trueque con el banco del tiempo, la moneda social y el centro social autogestionado. Esta espacialidad funcional sólo está levemente insinuada en las prácticas identificadas en Valladolid. La ausencia de redes densas y el grado de alternativa de la base social -los condicionantes que el contexto impone- no hacen posible un camino que permita en la práctica un grado de ruptura significativo con el capitalismo en torno a conceptos nucleadores como lo local y la proximidad.

Las cualidades y la dimensión del espacio producido difieren notablemente en función de cada práctica. En algunas, como el Banco del Tiempo o la moneda social Vecino difícilmente se puede argumentar que tienen o han tenido en la ciudad de Valladolid esa capacidad. Tampoco las redes de consumo. El número de personas que las integran, medido en capacidad de demanda de productos, no es suficiente para mantener una cifra significativa de superficie dedicada al cultivo agroecológico en las inmediaciones de la ciudad. Aquélla no pasa de unas pocas hectáreas y los escasos productores que, comprometidos con la filosofía de las redes y con el civismo ecológico (Seyfang, 2006), han visto en ellas una oportunidad emprende- 
dora, observan cómo sus cuentas de resultados se mantienen en un equilibrio inestable. El contacto entre objetos cercanos, en la terminología de Miller (2004), - productores y consumidores vinculados por un mismo convencimiento- no ha tenido la oportunidad de alterar la morfología del espacio periurbano.

Si bien en sentido opuesto, no ocurre lo mismo con los huertos comunitarios y los centros sociales autogestionados. Los primeros nacen con la voluntad de proyectar sus propiedades hacia el exterior. Salvo el caso del Gerihuerto de Parquesol -ubicado en el interior de una residencia de la tercera edad después de un intento fallido de ocupación de una parcela municipal vacía- y de Entrehuertos -en el interior de un huerto ocupacional promovido por el Ayuntamiento de Valladolid-, los demás han renunciado, incluso activamente, a la existencia de cualquier límite o barrera entre el huerto y el entorno. Se concibe la huerta como un espacio permeable. Los vecinos, no sólo los «hortelanos», pueden transitar por ella con normalidad, recoger sus frutos, compartir conocimientos, participar en las dinámicas sociales que allí se desarrollan y disfrutar de la experiencia reparadora que se origina en torno al trabajo de la tierra.

Los huertos urbanos están dotados de una estética particular. No es sólo la aparición de un nuevo paisaje dentro del conjunto edificado, sino la utilización de materiales de desecho, enseres y utillaje en desuso de una forma creativa. Los huertos urbanos vinculados a las prácticas económicas alternativas son un buen ejemplo de economía circular además de que toda alternativa posee también su estética alternativa.

Se puede reconocer un variado repertorio de centros sociales autogestionados (Martínez, 2002). La casuística se resuelve en Valladolid en tres posibilidades: la de carácter transgresor en lo cultural (Las Dagas), la de reflexión política (Vientos del Pueblo) y la que a estos dos rasgos une el compromiso social con el entorno más inmediato -el barrio- y con la construcción de un especial espacio interior. Es el caso del CsA La Ortiga. Este comparte con otras iniciativas la crítica al sistema capitalista, la ruptura de las convenciones de género y raza y el feminismo activo. Con estos elementos construye un «espacio liberado» que tiene como recinto el interior del local en el que se ubica y en el que la proximidad es incompatible con la indiferencia hacia el otro. En la entrevista realizada aparece con reiteración una noción que se vuelve nuclear: el cuidado mutuo. Sin duda, la geografía más autónoma de todas las estudiadas en la ciudad de Valladolid. 


\section{CONCLUSIONES}

Las prácticas económicas alternativas conocen un desarrollo acusado en la ciudad de Valladolid a partir del año 2012, tanto por el número de iniciativas que se ponen en marcha como por el volumen de personas que comienzan a reunir. No parece que el impacto de la crisis económica sobre el empleo condicione su desarrollo, magnitud y evolución, pero sí que están influidas por la crisis política, social y de valores que hace emerger. Salvo la moneda social Vecino, las prácticas no resuelven necesidades generadas por el impacto de la crisis ni atienden a grupos en situación de especial vulnerabilidad. De hecho, el perfil de los actores se aleja notablemente de esas coordenadas. Los participantes comparten convencimientos personales y discursos relativamente comunes. Estos se explicitan en cada práctica y, sobre todo, parecen ganar en densidad social en aquellas de espacialidad más cercana, como es el caso de los huertos comunitarios y los centros sociales autogestionados. No obstante, la base social de las prácticas no está marcada por su personalidad contrahegemónica. Se reconocen los profundos desequilibrios del sistema, pero se trabaja por limar alguna de sus aristas. Se produce así un fuerte desacople entre el potencial transformador que, desde un punto de vista teórico, contiene cada una de las prácticas y su práctica concreta en la ciudad.

El significado económico de las PEA en la ciudad de Valladolid es reducido, ya que la entidad de los flujos que generan conforma circuitos económicos de pequeña magnitud. Una reflexión semejante se puede hacer del espacio que construyen. Este no deja de ser un contrapunto marginal al generado por los actores principales que operan en la producción del espacio urbano; pero posee la cualidad de hacer equiparables continente y contenido. Uno es una prolongación del otro: espacios abiertos, autónomos y sostenibles que se ensamblan con la autogestión, la proximidad personal y la deliberación en la toma de decisiones.

Si bien es necesario indagar en la condición transformadora de lo local, las PEA se han entendido como una respuesta al modelo capitalista económico imperante, como una formulación que pretende superarlo poniendo el acento en la cercanía y la proximidad. Los convencimientos personales que implica su participación en ellas hacen que en no pocas ocasiones ese carácter de respuesta sea difícilmente cuestionable; pero su validez como alternativa transformadora está directamente relacionada con su escala. En ésta desempeña una función relevante el contexto espacial, social e histórico. En ciudades medias como la que se presenta, con una historia social, 
política y urbana reciente marcada por la sedimentación de valores conservadores, las PEA no pueden ser entendidas como la construcción de un nuevo espacio económico. Son fundamentalmente un espacio social y político. Un espacio en el que prima la cercanía y la comunidad, en el que se combate la fragmentación cultural del espacio urbano inherente a la ciudad moderna, en el que se intentan romper las barreras que los valores antes enunciados imponen. Un espacio en el que se produce una relectura del tradicional compromiso de los sectores más movilizados de la sociedad con una visión progresiva de la ciudad y de la socialización que es sustancial a ella. De hecho, se puede afirmar que su valor estriba más en la experiencia social y política que llevan implícita que en la práctica económica que las sustentan.

El enfoque contextualizado de este estudio ha permitido identificar un tejido de proyectos sociales alternativos susceptible de densificarse a través de redes interconectadas más allá de la escala local. Investigar en qué medida estas prácticas pueden integrarse de forma articulada en visiones más amplias y analizar su potencial transformador a mayor escala, plantea la necesidad de seguir ampliando el conocimiento de experiencias similares en otras ciudades españolas, para indagar además en la posible existencia de trayectorias análogas o fortalezas desiguales. Resulta también fundamental profundizar en los factores, todavía escasamente conocidos, que explican la emergencia de prácticas alternativas en determinadas áreas de la ciudad, así como valorar su capacidad para participar de forma activa en la formulación de enfoques diferentes para la agenda de las políticas públicas sociales, urbanas y territoriales.

\section{BIBLIOGRAFÍA}

Abad, Jaime \& Abad, Mercedes (2014). La economía social y solidaria como alternativa económica. Bienes comunes y democracia. Recerca. Revista de Pensament i Anàlisi, 15, 55-75.

Bioteau, Emmanuel \& Fleuret, Sébastien (2014). Quelques jalons pour une gèographie de l'economie sociale et solidaire. Annales de Gèographie, 697, 890-911. doi:10.3917/ag.697.0890

Bresnihan, Patrick \& Byrne, Michael (2015). Escape into the City: Everyday Practices of Commoning and the Production of Urban Space in Dublin. Antipode, 47(1), 36-54. doi:10.1111/anti.12105 
Buttle, Martin (2008). Diverse economies and the negotiations and practices of ethical finance: the case of Charity Bank. Environment and Planning, 40, 2097-2113. doi: 10.1068/a39317

Cardoso, Gustavo \& Jacobetty, Pedro (2013). Navegando la crisis: culturas de pertenencia y el cambio social en red. En Castells, Manuel, Caraça, Joao \& Cardoso, Gustavo (eds). Después de la crisis (245-285). Madrid: Alianza Editorial.

Conill, Joana, Cárdenas, Amalia, Castells, Manuel, Hlebik, Svetlana \& Servon, Lisa (2012). Otra vida es posible: prácticas económicas alternativas durante la crisis. Barcelona: Ediciones UOC.

Esquirol, Josep María (2015). La resistencia íntima. Ensayo de una filosofía de la proximidad. Barcelona:Acantilado.

Felber, Christian \& Hagelberg, Gus (2017). The economy of common good. A Workable, Transformative Ethics-Based Alternative. The Next System Project.

Fernàndez,Anna \& Miró Ivan (2016). L'economia social $i$ solidària a Barcelona. Barcelona:Ajuntament de Barcelona.

Fickey, Amanda \& Hanrahan, Kelsey, (2014). Moving Beyond Neverland: Reflecting Upon the State of the Diverse Economies Research Program and the Study of Alternative Economic Spaces. ACME: An International E-Journal for Critical Geographies, 13 (2), 394-403.

García, José Luis (2000). De la urgencia social al negocio inmobiliario. Promoción de viviendas y desarrollo urbano en Valladolid (19601992). Valladolid: Secretariado de Publicaciones de la Universidad de Valladolid.

García, Jordi (2013). Economía solidaria: otra economía para otro desarro1lo. En Retos y futuro del desarrollo económico local, 185-194. Madrid: Red de Entidades para el Desarrollo Local (REDEL).

García,Antonio, Fernández, Víctor, Caravaca, Inmaculada \& González, Gema (2016).Actividades creativas, transformaciones urbanas y paisajes emergentes. El caso del casco norte de Sevilla.Documents d'Anàlisi Geografia, 62/1, 27-54. doi:10.5565/rev/dag. 245

Gibson, Katherine \& Graham, Julie (2008). Diverse economies: performative practices of 'other worlds'. Progress in Human Geography, 32(5), 613-632. doi:10.1177/0309132508090821

Gonick, Sohpie (2016). Indignation and inclusion: activism, difference, and emergent urban politics in postcrash Madrid. Environment and Planning, 34(2), 209-226. doi: 10.1177/0263775815608852 
Gonzalo, Constantino (2010). Una visión global del movimiento asociativo vecinal regional durante la transición: 1970-1986. Estudios Humanísticos, 698, 195-220.

- (2011). El movimiento vecinal como modelador del urbanismo: el caso de Valladolid en la Transición y la campaña «La Ribera es nuestra». Studium. Revista de Humanidades, 17, 45-52.

Gritzas, Giorgos \& Kavoulakos, Karolos Iosif (2015). Diverse economies and alternative spaces: An overview of approaches and practices. European Urban and Regional Studies, 23(4), 917-934. doi:10.1177/0969776415573778

Healey, Stephen (2009). Economies, Alternative. En Kitchin, Rob \& Thrif, Nigel (eds.). International Encyclopedia of Human Geography. vol I., 338-344.

Jones,Andrew \& Murphy, James Thomas (2011). Theorizing practice in economic geography: Foundations, challenges and possibilities. Progress in Human Geograpby, 35(3), 366-392. doi:10.1177/0309132510375585

Leyshon, Andrew, Lee, Roger \& Williams, Colin (eds) (2003). Alternative economic spaces. Londres: Sage.

Martínez, Miguel (2002). Okupación de viviendas y de centros sociales. Barcelona: Virus.

Mattei, Ugo (2013). Bienes comunes: un manifiesto. Madrid:Trotta.

Méndez, Ricardo (2015). Redes de colaboración y economía alternativa para la resiliencia urbana: una agenda de investigación. Biblio 3 W. Revista Bibliográfica de Geografía y Ciencias Sociales. 25 de noviembre de 2015, XX(1-139), 1-23.

Michelini, Juan José, Méndez, Ricardo \& Abad, Luis (2017). Movilización social y alternativas alimentarias en áreas urbanas. Los grupos de consumo agroecológico en Madrid. Ciudad y Territorio - Estudios Territoriales, 194, 679-698.

Miller, Harvey (2004). Tobler's First Law and Spatyal Analisys. Annals of the Association of American Geographers, 94, 284-289. doi:10.1111/j.1467-8306.2004.09402005.x

Moulaert, Frank, Parra, Constanza \& Swyngedouw, Erik (2014). Ciudades, barrios y gobernanza multiescalar en la Europa urbana. Eure, 40(119), 5-24.

Nel·lo, Oriol (2015). La ciudad en movimiento. Crisis social y respuesta ciudadana. Barcelona: Díaz \& Pons. 
North, Peter (2005). Scaling alternative economic practices? Some lessons from alternative currencies. Transactions of the Institute of British Geographers, 30, 221-233. doi: 10.1111/j.1475-5661.2005.00162.x

Paddeu, F. (2012). Faire face à la crise économique à Detroit: les pratiques alternatives au service d'une résilience urbaine? L'Information Géographique, 76(4), 119-139.

Psarikidou, Katerina (2015). Rethinking innovation through a moral economy lens: the case of alternative agro-food and mobility practices. Ephemera. Theory \& Politics in Organization, 15, 67-93.

Ruiz, Ana (2013). Urbanismo y participación ciudadana: el caso de La Rondilla en el Valladolid de los años setenta. Estudios Geográficos, LXXIV (275), 611-637.

Sánchez, José Luis (2017). Las prácticas económicas alternativas en perspectiva geográfica. Salamanca: Universidad de Salamanca.

Sánchez, José Luis, Nicolás, Adrián, Alonso, José Luis \& Moro, Lourdes (2017). Regeneración urbana, innovación social y prácticas económicas alternativas en ciudades medias: el barrio del Oeste (Salamanca), Ería, 1 (nueva época), 67-82. doi:10.17811/er.1.2017.67-82

Seyfang, Gill (2006). Ecological citizenship and sustainable consumption. Examining local organic food networks. Journal of Rural Studies, 22, 383-395. doi:10.1016/j.jrurstud.2006.01.003

Spencer, Gregory M. (2015). Knowledge Neighbourhoods: Urban Form and Evolutionary Economic Geography. Regional Studies, 49 (5), 883-898. doi: 10.1080/00343404.2015.1019846

Tornaghi, Clara (2014). Critical geography of urban agriculture. Progress in Human Geograpby, 38(4), 551-567. doi: 10.1177/0309132513512542

Vanolo, Alberto (2013). Alternative Capitalism and Creative Economy: the case of Christiana. International Journal of Urban and Regional Reserarch, 37(5), 1785-1798. doi:10.1111/j.1468-2427.2012.01167.x

Watts, David, Ilbery, Brian \& Maye, Damian (2005). Making reconnections in agro-food geography: alternative systems of food provision. Progress in Human Geography, 29(1), 22-40. doi:10.1191/0309132505ph526oa 